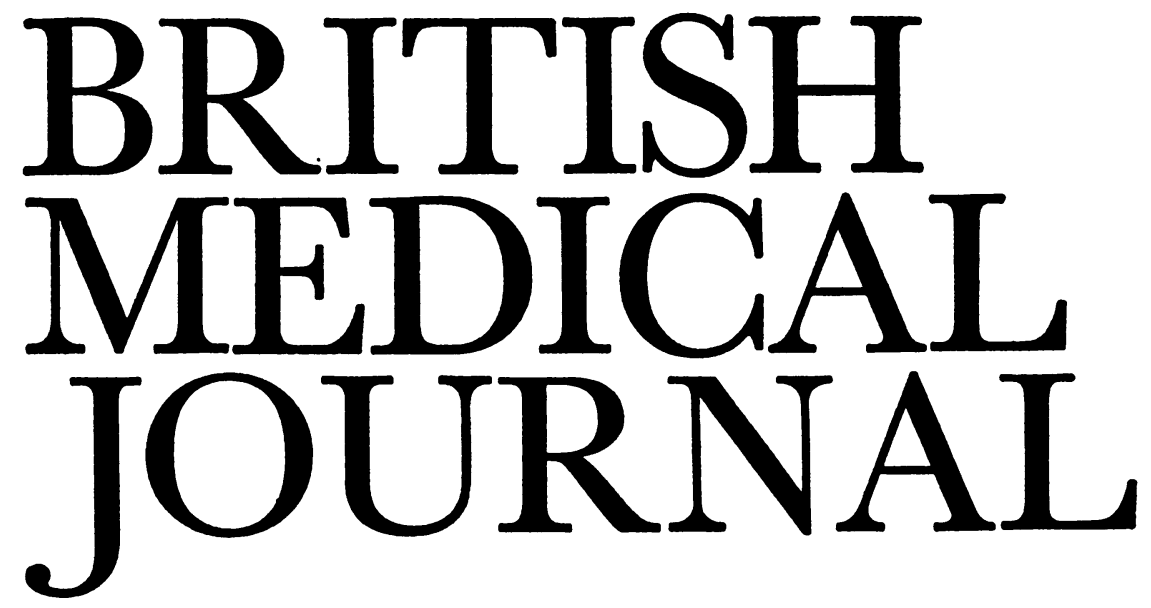

LONDON SATURDAY 24 MARCH 1973

\title{
Premenstrual Symptoms
}

Shortly before or during menstruation women and girls are more liable than usual to fail examinations, absent themselves from work, experience admission to hospital, develop acute psychiatric symptoms, commit crimes, attempt suicide, be involved in accidents, or die by accident or suicide. ${ }^{1}$ More than one woman in nine reports severe degrees of pain, irritability, or headache in association with her periods; one woman in 16 reports that she gets depressed or tense. Four out of five women are conscious of swelling of the body, one in 14 restricts her activities during menstruation, and one in seven has irregular periods. ${ }^{2}$

In a study of healthy young women $39 \%$ had troublesome premenstrual symptoms, $54 \%$ passed clots in their menstrual flow, no less than $70 \%$ had a cyclical localized acneiform eruption, usually on the face, and only $17 \%$ were free from pain during menstruation. ${ }^{3}$ Only a fraction of women with menstrual disorders bring their symptoms to their doctor and of those who do few are passed to the gynaecologist or the psych-atrist. In the absence of pathological lesions there are two common but distinct entities-the premenstrual syndrome and dysmenorrhoea.

The premenstrual syndrome consists of nervous tension, irritability, anxiety, depression, bloated feelings of the abdomen and breasts, swelling of the fingers and legs, tightness and itching of the sk.n with or without skin eruptions, headaches, dizziness, and palpitations. Less commonly there occur hypersomnia, excessive thirst and appetite, increased sex desire, and in some women an increased tendency for asthma, migraine, vasomotor rhinitis, urticaria, and epilepsy. ${ }^{4}$ These symptoms may be accompanied by abdominal pain or cramps, which always begin before and usually subside with the onset of flow. Symptoms commonly begin two to 12 days before menstruation and in the majority of patients are relieved at the onset of menstruation. Many sufferers experience a remarkable sense of wellbeing during pregnancy only to experience a recrudescence of symptoms after the puerperium.

A variety of aetiological theories have been examined but none substantiated. In particular the conviction that retention of water and sodium causes the symptoms persists, and symptomatic relief has been reported with salt and water restriction and the use of simple diuretics seven to ten days premenstrually. Unfortunately carefully controlled studies have shown no clear relationships between symptoms and water and sodium retention, which frequently occurs in midcycle, when symptoms are uncommon. ${ }^{5} \mathrm{~K}$. Dalton ${ }^{6}$ and others have advocated giving progesterone $25-100 \mathrm{mg}$ by injection or $100-400 \mathrm{mg}$ suppositories daily from mid-cycle to menstruation. This treatment is often effective, but oral progestogens are less predictable. Many patients experience relief on an oral contraceptive, though much trial and error may be required to find the most suitable preparation. But before venturing on hormonal treatment it is worth attemoting symptomatic treatment with diazepam or chlordiazepoxide.

The premenstrual syndrome spans a wide age range. It may even persist after the menopause and is unaffected by age or parity, and sufferers score highly on tests of neuroticism. In contrast, primary or spasmodic dysmenorrhoea occurs in young women, has no correlation with neuroticism or premenstrual symptoms, and tends to decline with age and parity. ${ }^{7}$ The pain, which is spasmodic or colicky, develops and is most severe on the first day of menstruation, though it may continue for two or three days.

Psychotropic drugs have no place in the treatment of primary dysmenorrhoea, and though amphetamines function both as an antispasmodic and a euphoriant their prescription for cyclical pain carries a serious risk of dependence and abuse. Pethidine, morphine, and other narcotics carry a similar risk, and simple analgesics such as aspirin or paracetamol are preferable. Buscopan (hyoscine-N-butylbromide) 10-20 mg three to four times daily or compound tablets of belladonna, ergotamine, and phenobarbitone one or two thrice daily are often effective in relieving the colicky pain ${ }^{8}$ If the pain is disabling and a spell of freedom is imperative the contraceptive pill combining a synthetic oestrogen and progestogen will usually offer relief, but the risks to a young woman need to be balanced.

Menstrual irregularity correlates with both premenstrual syndrome and emotional instability, while in many normal women amenorrhoea may be precipitated by relatively trivial psycholog:cal events such as leaving home to enter a residential nursing school or college. 9 More severely stressful events such as air raids or fear of extermination lead to a 
high incidence of amenorrhoea. In most cases the menses spontaneously recur within a few months unless some other factor such as malnutrition is also operating. ${ }^{2}$ Fear of being pregnant commonly delays the onset of menstruation, but less often a desperate desire for pregnancy or a phantasied love relationship may lead to a pseudocyesis or phantom pregnancy. The woman is convinced that she has conceived and develops many signs and symptoms of maternity. Amenorrhoea is common, but not invariable. Abdominal swelling, usually accompanied by lumbar lordosis, the exper-ence of fetal movements, breast soreness, swelling and secretion, and morning sickness complete the picture. But on examination no fetal parts can be felt, the fetal heart not heard, and rectal examination discloses a uterus of normal size. Though there is often evidence of a hysterical personality and low intelligence, this is not always so.

In anorexia nervosa amenorrhoea is a constant feature and may precede the abnormal attitudes to eating and body weight. The uniformity of some aspects of the syndrome is striking and lends support to the view that it is a specific endocrine disorder. G. F. M. Russell ${ }^{5}$ has adduced biochemical evidence in support of this. However, in terms of cl nical management anorexia nervosa is best regarded as a chronic, recurrent, and often severe neurotic disorder in which physical and psychological methods of treatment need to be combined.

The relationship between the neuroendocrine system and emotion is complex, and despite advances in understanding 10-11 further basic research needs to be done. Meanwhile much can be done to help women live with their cyclical changes.

1 Tonks, C. M., British fournal of Hospital Medicine, 1968, 1, 383.

2 Kessel, N., and Coppen, A., Lancet, 1963, 2, 61.

- Sutherland, H., and Stewart, I., Lancet, 1965, 1, 1180

- Rees, L., fournal of Mental Science, 1953, 99, 62.

Rees, L., Fournal of Mental Science, 1953, 99, 62.

- Russell, G. F. M., Fournal of Psychosomatic

7 Dalton, K., Practitioner, 1971, 206, 681.

'Tindall, V., R., British Medical fournal, 1971, 1, 329.

- Hain, J. D., Linton, P. H., Eber, H. W., and Chapman, M. M., fournal of Psychosomatic Research, 1970, 14, 81.

10 Beaumont, P. J. V., in Psychiatric Aspects of Medical Practice, ed. B. M Mandlebrote, and M. G. Gelder, p. 222. London, Staples Press, 1972 11 Loraine, J. A., and Bell, E. T., Fertility and Contraception in the Human Female. Edinburgh, E. \& S. Livingstone, 1968.

\section{Proposal for an Academy}

In our columns this week a group of distinguished signatories to a letter (page 737) examine the role of the royal colleges and allied bodies in relation to Britain's entry into the European Economic Communi'y, and propose the creation of a British academy of medicine. Since about 1950 the colleges have increasingly taken on the function of advancing education in the branches of medicine represented in them. As the letter puts it, a college reoresents the interests of its discipline and not that of individual practitioners. This is a notable and recent shift of emphasis. What is now proposed therefore is that the distinctive contributions which each college makes in its sphere should be brought together in an academy, in which all the existing colleges and faculties in Great Britain and Ireland would be represented.

As well as being a valuable source of information and advice to the E.E.C. countries on the British way of medical life such a body might help the constituent colleges themselves by providing a forum for the discussion of common problems. The generality of medicine, say the authors of this letter, cries out for a unifying, co-ordinating force, and this general aspiration undoubtedly reflects the particularities of practice and research. Clinical work is increasingly a matter for a team rather than an individual, and research is thought to be hardly worthy of the name unless it is multidisciplinary or at least interdisciplinary. So that we see the paradox of increasing collaboration at ground level, yet increasing dispersal in the sky. The B.M.A. itself is familiar enough with this situation. Could an academy succeed among such contentious people as doctors show themselves to be in public debate?

The sponsors of the new venture are not wedded to the title academy, and it would probably prevent confusion if they adopted another. An academy is usually thought of as an educational institution or a society devoted to the pursuit of learning. But what Sir Thomas Holmes Sellors and his colleagues seem to have in mind is a co-ordinating commitlee. Nothing could be worse than the creation of a pseudoacademy embellished with the mummery of gold chains, maces, gavels of exotic wood, and hypnotic after-dinner speeches. If it is judged by its declared object of providing an opportunity for the colleges and faculties to profit from each other's experience and to present a clear and united opinion on matlers of major policy, then the proposed new body will find itself among several others with similarly unitary but in various ways differently directed aims. Among them are the B.M.A., the (presumably reconstituted) G.M.C., and (with only slight broadening of its representation) the Joint Consultants Committee. In addition, as the letter itself mentions, medical education finds expert expression in the Conference of Postgraduate Deans. Yet when all that is said, the present proposal does offer something different. It therefore deserves examination and debate. But if the sponsors really believe it "would require no substantial funds," the reader must be forgiven for wondering how seriously they have thought about the academy's role in today's expensive world. The G.M.C. is one comparable body that could offer advice on that.

\section{Serum Cholesterol in Children}

Prospective studies in adults have now shown that a high serum cholesterol concentration is one of the most readily identifiable risk factors in the development of coronary heart disease. ${ }^{1}$ It is not yet known with certainty whether lowering the serum cholesterol will prevent or delay the onset of this disease, but the results of a recent dietary trial in Finland ${ }^{2}$ are promising. Because there is considerable evidence that atherosclerosis may originate in childhood ${ }^{3}$ it would seem desirable that risk factors should be identified as early in life as possible, so that preventive measures can be introduced at a stage when they are most likely to influence the underlying pathological process.

At present it is not clear whether serum lipid levels in adults can be predicted from estimations made during infancy and childhood. Familial hyperbetalipoproteinaemia is probably the most common of the genetically determined hyperlipidaemias and can usually be diagnosed in childhood, at least after the first year of life. ${ }^{4}$ But exact information on 